\title{
Simultaneous and Sequential Integration by Cre/loxP Site-Specific Recombination in Saccharomyces cerevisiae
}

\author{
Ho-Jung Choi and Yeon-Hee Kim* \\ Biomedical Engineering and Biotechnology Major, Divison of Applied Bioengineering, Dong-Eui University, Busan 47340, Republic of Korea
}

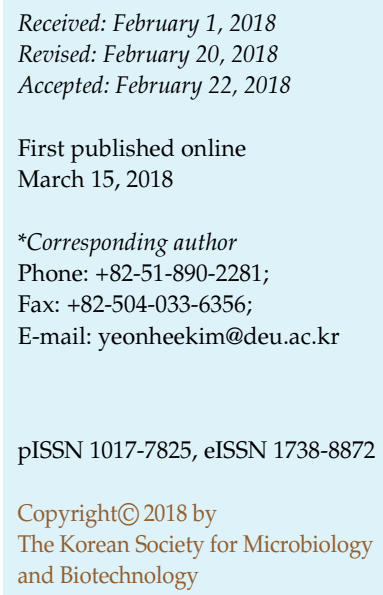

A Cre/loxp- $\delta$-integration system was developed to allow sequential and simultaneous integration of a multiple gene expression cassette in Saccharomyces cerevisiae. To allow repeated integrations, the reusable Candida glabrata MARKER (CgMARKER) carrying loxP sequences was used, and the integrated $C g M A R K E R$ was efficiently removed by inducing Cre recombinase. The XYLP and XYLB genes encoding endoxylanase and $\beta$-xylosidase, respectively, were used as model genes for xylan metabolism in this system, and the copy number of these genes was increased to 15.8 and 16.9 copies/cell, respectively, by repeated integration. This integration system is a promising approach for the easy construction of yeast strains with enhanced metabolic pathways through multicopy gene expression.

Keywords: $\delta$-Sequence integration, Cre/loxP system, Candida glabrata MARKER, simultaneous multiple integration
Saccharomyces cerevisiae plays a key role in industries ranging from pharmaceutical production to ethanol and biomass production and is an important industrial microorganism. In all of these applications, the ability to introduce and maintain stable foreign genes is extremely important because large-scale production of foreign genes in recombinant yeast often uses complex medium. Highcopy-number plasmids derived from YEp are often mitotically unstable, and selective conditions are required for the maintenance of these plasmids. However, YEp vectors may be unstable at high expression even in selective conditions [1,2]. Therefore, direct integration of a target gene into the host chromosome is an effective method for the stable expression of foreign genes in S. cerevisiae [3]. However, traditional integration methods using mitotically stable vectors such as YIp have limitations regarding the number of genes that can be inserted, low copy number, and regulation of the integration process. To increase the integration copies of a target gene, repetitive chromosomal DNA sequences such as $\delta$-sequences [4-7] and rDNA $[8,9]$ have been used. In general, $\delta$-sequences were more versatile than rDNA sequences, and high-level expression of cloned genes using a sequential $\delta$-integration method was reported
[3]. For repeated and sequential integration, incorporation of a reusable selective marker and $\delta$-sequence-mediated integration are necessary. In the original $\delta$-integration method, the hisG-URA3-hisG cassette was used as a reusable selective marker, but it was impossible to simultaneously integrate multiple genes. In a previous study, we developed a PCR-mediated chromosome splitting method combined [10] with the Cre/loxP site-specific recombination system for marker rescue and repeatedly split chromosomes [11, 12]. Using this system, we attempted introduction of a reusable selective marker system for the sequential and/or simultaneous integration of multiple genes by $\delta$-sequencemediated integration.

In the present study, two enzymes (endoxylanase and $\beta$-xylosidase) required for degradation of xylan were used as model enzymes, and integrative plasmids harboring the Candida glabrata MARKER (CgMARKER) carrying the loxP sequences and $\delta$-sequence were constructed. $S$. cerevisiae

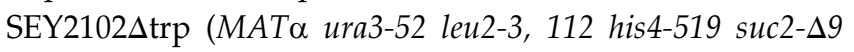
$\operatorname{trp} 1:: K a n M X)$ was used as the host strain, and the XYLP and $X Y L B$ genes encoding endoxylanase and $\beta$-xylosidase, respectively, were stably integrated into the yeast chromosome by $\delta$-sequence-mediated integration. The YPD nutrient 
medium and synthetic complete (SC) medium for S. cerevisiae and cultivation methods have been described in a previous report [11]. YPDG (YPD containing 1\% galactose) medium was used for galactose-inducible gene expression. 5-Fluoroorotic acid (5-FOA) medium was used for removal of the pSH47 plasmid containing the URA3 selective marker [11]. The methods for yeast transformation, pulsed-field gel electrophoresis (PFGE), Southern hybridization, and thin-layer chromatography were previously described by Kim et al. $[13,14]$. The enzyme activities of endoxylanase and $\beta$ xylosidase were measured by respective assay methods $[15,16]$.

The $\mathrm{pRS} \delta$ - $\mathrm{T}$ and $\mathrm{pRS} \delta$ - $\mathrm{H}$ plasmids were constructed for integration of the $X Y L P$ and $X Y L B$ genes, respectively, into the $\delta$-sequence of the yeast chromosome. We used the YFLWdelta4 sequence (332 bp) as the $\delta$-sequence because it shares high homology $(>90 \%)$ with other $\delta$-sequences [17]. The YFLWdelta4 sequence was cloned into pRS306 (a YIp vector), resulting in the pRS $\delta$ plasmid. For repeated integration by introduction of a reusable selective marker, the loxP-CgTRP1-loxP (1.2 kb) and loxP-CgHIS3-loxP (1.7 kb),
Candida glabrata TRP1, and HIS3 cassette carrying the loxP sequences ( $34 \mathrm{bp}$ ) were cloned into the pRS $\delta$ plasmid, resulting in the pRS $-\mathrm{T}(6 \mathrm{~kb})$ and pRS $\delta-\mathrm{H}(6.5 \mathrm{~kb})$ plasmids, respectively. The CgTRP1 and CgHIS3 genes $(72 \%$ and $64 \%$ nucleotide sequence identity with the corresponding $S$. cerevisiae genes, respectively) were used to avoid undesirable recombination between the integrative plasmids and the marker loci within the $S$. cerevisiae genome. Finally, the $X Y L P$ and $X Y L B$ gene expression cassettes, GAL10p-MF $\alpha$ s.s-XYLP-Gal7t and GAL10p-MFa s.s-XYLB-Gal7t from the pGMF $\alpha-X y l P$ [18] and pGMF $\alpha-X y l B$ plasmids, respectively, were inserted into the $\mathrm{pRS} \delta$ - $\mathrm{T}$ and $\mathrm{pRS} \delta$ - $\mathrm{H}$ plasmids, resulting in the $\mathrm{pRS} \delta$-XylP and $\mathrm{pRS} \delta$-XylB plasmids.

pRS $\delta$-XylP was linearized by digestion with XhoI, which is a unique restriction site within the $\delta$-sequence, and introduced into the SEY2102Atrp strain. Transformants expressing endoxylanase were selected on tryptophandeficient SC plates, and one transformant that showed endoxylanase activity by Congo-red staining was designated the $\delta$-P strain (Fig. 1A). The endoxylanase was efficiently secreted into the medium by mating factor $\alpha$ signal sequence

A Integration of endoxylanase expression cassette at the $\delta$-sequence : transformation of linearized pRSo-XylP plasmid into SEY2102 $\Delta$ trp

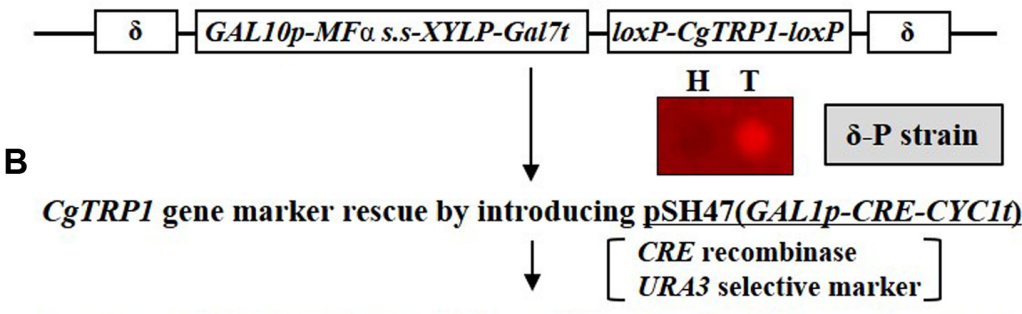

Remove pSH47 plasmid on 5-FOA medium after $C g T R P 1$ marker rescue

C

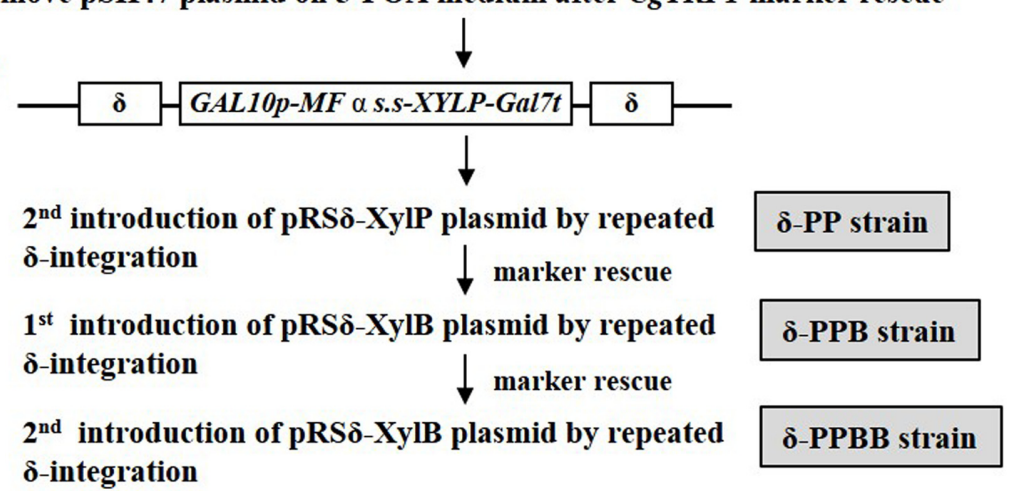

Fig. 1. Procedure for $\delta$-sequence-mediated integration of the XYLP and XYLB gene expression cassette and construction of yeast strains with endoxylanase and $\beta$-xylosidase activities.

The endoxylanase activity was determined by Congo-red assay. $\mathrm{H}$ and T indicate the SEY2102 2 trp host strain and $\delta$-P strain, respectively. MF $\alpha$ s.s: mating factor $\alpha$ signal sequence of S. cerevisiae; CgTRP1: TRP1 gene of Candida glabrata; loxP: ATAACTTCGTATAATGTATGCTATACGAAGTTAT (for sequential integration). 
A

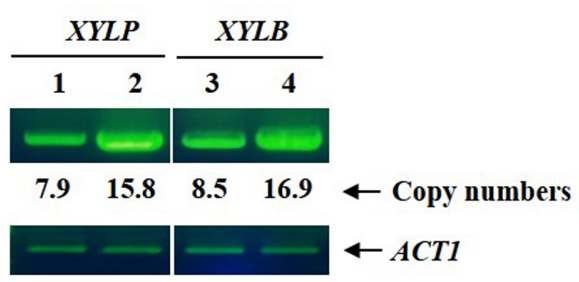

B

\begin{tabular}{|c|c|c|c|}
\hline Strains & $\begin{array}{c}\text { Cell growth } \\
\left(\mathbf{O D}_{600}\right)\end{array}$ & $\begin{array}{c}\text { Endoxylanase } \\
\text { activity (unit/ml) }\end{array}$ & $\begin{array}{c}\beta \text {-xylosidase } \\
\text { activity (unit/ml) }\end{array}$ \\
\hline SEY2102 $\Delta$ trp & 27.9 & - & - \\
\hline $\boldsymbol{\delta}-\mathbf{P}$ & 30.5 & $8.3 \pm 0.01$ & - \\
\hline$\delta-\mathbf{P P}$ & 28.6 & $12.5 \pm 0.03$ & - \\
\hline ठ-PPB & 24.6 & $12.1 \pm 0.02$ & $0.5 \pm 0.01$ \\
\hline ठ-PPBB & 25.1 & $13.2 \pm 0.05$ & $0.87 \pm 0.01$ \\
\hline$\delta$-PB & 26.3 & $9.5 \pm 0.07$ & $0.62 \pm 0.01$ \\
\hline
\end{tabular}

Fig. 2. Analysis of the copy numbers of endoxylanase and $\beta$ xylosidase genes using RT-PCR (A) and comparison of cell growth $\left(\mathrm{OD}_{600}\right)$, and endoxylanase and $\beta$-xylosidase activities in each strain (B).

Total RNA was isolated from each transformant, and cDNA was synthesized from total RNA by RT-PCR. The XYLP, XYLB, and ACT1 (internal control) genes were amplified by PCR using each cDNA as template, and the products were visualized by agarose gel electrophoresis. Lane 1: $\delta$-P strain; lane 2: $\delta$-PP strain; lane 3: $\delta$-PPB strain; lane 4: $\delta$-PPBB strain.

(MFas.s). For sequential integration, the CgTRP1 selective marker of the $\delta$-P strain was removed by introducing the pSH47 plasmid (GAL1p-CRE-CYC1t). The Cre recombinase was expressed in YPDG medium for induction of galactose, and a $\delta$-P $\Delta$ trp cell that had lost the CgTRP1 gene by excisional recombination between the two direct loxP repeats was selected. The pSH47 plasmid harboring the URA3 gene as a selective marker was also cured by selection and spreading onto YPD medium containing 5-FOA (Fig. 1B). The goal of employing the loxP-CgMARKER-loxP selection cassette was to allow sequential and simultaneous integrations of the same or different genes via $\delta$-integration. Therefore, the pRS $\delta$-XylP plasmid was retransformed into the $\delta$-P $\Delta$ trp strain by the same procedure, and the $\delta$-PP strain was also constructed (Fig. 1C). Subsequently, pRS $\delta$ $\mathrm{XylB}$ was linearized by digestion with $\mathrm{XhoI}$ and introduced into the $\delta$-PP strain. Transformants with $\beta$-xylosidase activity were selected on histidine-deficient SC plates, and transformants showing $\beta$-xylosidase activity by two rounds of $\delta$-integration were designated the $\delta$-PРB and $\delta$-РРВB strains (Fig. 1C). Analysis of cell samples before and after CgMARKER curing by Southern hybridization and auxotrophic phenotype analysis confirmed that the CgTRP1 and CgHIS3 markers were efficiently removed $(>80 \%)$ from the integrated chromosomes by Cre recombinase (data not shown). To determine the copy number of integrated XYLP and XYLB genes, each strain was grown in YPDG medium, and the copy numbers were compared by RT-PCR and signal intensity analysis [18]. In the $\delta$-PP and $\delta$-PPBB strains, a 2 -fold increase in copy number was observed compared with the $\delta$-P and $\delta$-PPB strains, respectively, by additional integration. Finally, the copy number of integrated XYLP and $X Y L B$ genes was 15.8 and 16.9 copies/cell, respectively, in the $\delta$-PPBB strain (Fig. 2A). The activities of endoxylanase and $\beta$-xylosidase in the $\delta$-PP and $\delta$-РPBB strains were also increased approximately 1.6-1.7 fold compared with the $\delta$-P and $\delta$-PPB strains, respectively, (Fig. 2B), and the endoxylanase activity was stably maintained despite repeated marker rescue, cultivation, and transformation. The XYLP genes were intensively integrated in chromosomes VIII and $\mathrm{V}$ in the $\delta$-PP strain (Fig. 3A, lane 2), and the XYLB genes tended to be intensively integrated into chromosomes VIII, V, and XIV (Fig. 3B). The increased copy number was presumed to be caused by integration targeted to a new location, as described in Lee and Da Silva [19]. The integrated $X Y L P$ and $X Y L B$ genes were stably maintained in chromosomes in non-selective medium over 60 generations compared with the gene introduced as an episomal type, and $98 \%$ of endoxylanase activity was maintained in the $\delta$-PPBB strain (Fig. 3C), indicating that expression of the cloned gene did not significantly affect the structural stability. Furthermore, the endoxylanase and $\beta$-xylosidase produced in the integrants efficiently hydrolyzed xylan to xylose and xylooligosaccharides (xylobiose and xylotriose) (Fig. 4). For more efficient employment of the CgMARKER-

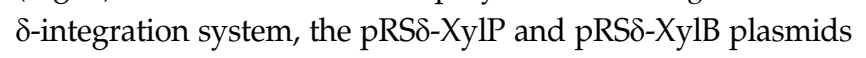
were simultaneously introduced into the SEY2102Atrp strain, and transformants were selected on tryptophan and histidine-deficient SC plates. A transformant showing endoxylanase and $\beta$-xylosidase activities was selected ( $\delta$-PB strain), and the enzyme activity was not significantly different from that of sequential integration (one round) (Fig. 2B). Moreover, the double selective markers were synchronously removed ( $>75 \%$ ) by inducing Cre recombinase (data not shown). These results demonstrated that the Cre/ lox $P$-mediated $\delta$-integration system was more versatile for 
A

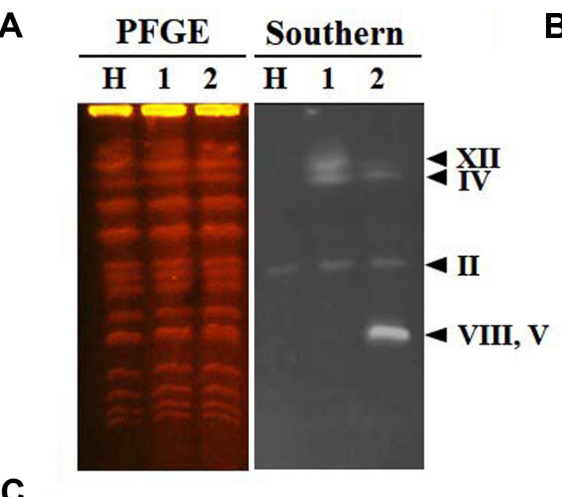

C

\begin{tabular}{ccccccc}
\hline \multirow{2}{*}{ Strains } & & \multicolumn{5}{c}{ Generations } \\
\cline { 3 - 7 } & & 0 & 10 & 20 & 40 & 60 \\
\hline \multirow{2}{*}{ SEY21024trp } & Mitotic stability (\%) & 100 & 80 & 76 & 72 & 68 \\
/pGMFa-Xylp & Residual activity (\%) & 100 & 85 & 81 & 75 & 65 \\
\hline \multirow{2}{*}{ o-PPBB } & Mitotic stability (\%) & 100 & 100 & 100 & 100 & 100 \\
& Residual activity (\%) & 100 & 100 & 100 & 99 & 98 \\
\hline
\end{tabular}

B PFGE $\frac{\text { Southern }}{\text { H } 344}$

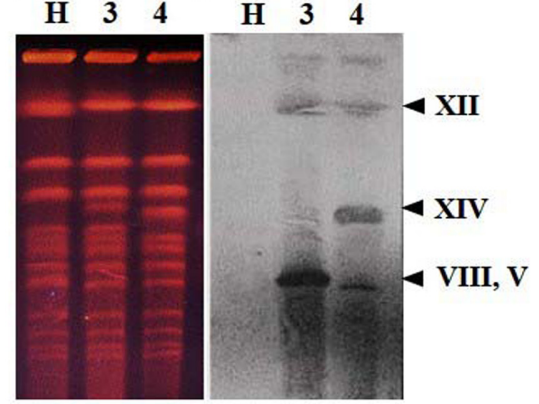

Fig. 3. Confirmation of the integration position by PFGE and Southern hybridization.

Gal10p (A) and XYLB genes (B) were used as probes. Lane H: SEY2102 $\Delta$ trp; lane 1: $\delta$-P strain; lane 2: $\delta$-PP strain; lane 3: $\delta$-PPB strain; lane 4: $\delta$ PPBB strain. Comparison of the mitotic stability and residual endoxylanase activity in the SEY2102 $\Delta$ trp/pGMF-Xylp (episomal type) and $\delta$-PPBB (integrative type) strains (C). These strains were cultivated for 60 generations in YPDG in a 50-ml baffled flask culture.

sequential and simultaneous integration of a gene expression cassette of two or more genes compared with the original $\delta$-integration system.

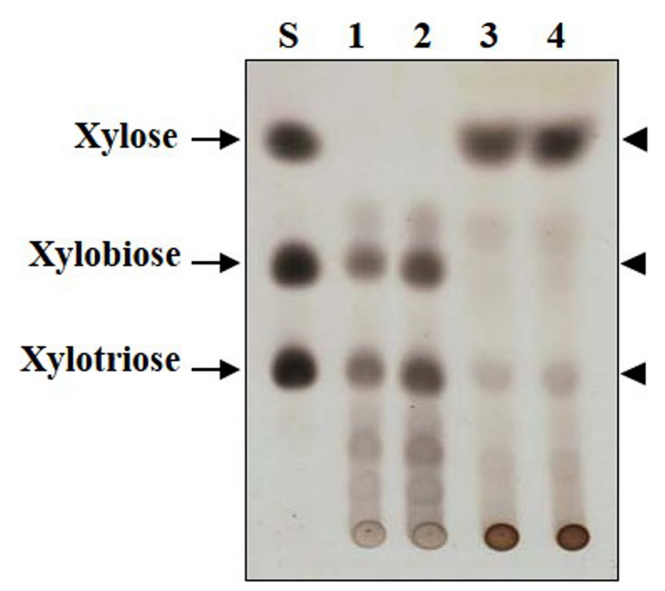

Fig. 4. Comparison of degradation products of xylan by thinlayer chromatography.

Each transformant was cultivated in YPDG medium for $48 \mathrm{~h}$, and $1 \%$ xylan (birchwood) was hydrolyzed by enzymes produced by each transformant. Lane S: standard mixture (xylose, xylobiose, and xylotriose); lane 1: $\delta$-P strain; lane 2: $\delta$-PP strain; lane 3: $\delta$-PPB strain; lane 4: $\delta$-PPBB strain.

\section{Conflict of Interest}

The authors have no financial conflicts of interest to declare.

\section{References}

1. Murray AW, Szostak JW. 1983. Pedigree analysis of plasmid segregation in yeast. Cell 34: 961-970.

2. Romanos MA, Scorer CA, Clare JJ. 1992. Foreign gene expression in yeast: a review. Yeast 8: 423-488.

3. Lee FW, Da Silva NA. 1997. Sequential delta-integration for the regulated insertion of cloned genes in Saccharomyces cerevisiae. Biotechnol. Prog. 13: 368-373.

4. Kim MD, Rhee SK, Seo JH. 2001. Enhanced production of anticoagulant hirudin in recombinant Saccharomyces cerevisiae by chromosomal $\delta$-integration. J. Biotechnol. 85: 41-48.

5. Sakai A, Shimizu Y, Hishimura F. 1990. Integration of heterologous genes into the chromosome of Saccharomyces cerevisiae using a $\delta$-sequence of yeast retrotransposon Ty. Appl. Microbiol. Biotechnol. 33: 302-306.

6. Mochizuki D, Miyahara K, Hirata D, Matsuzaki H, Hatano T, Fukui S, et al. 1994. Overexpression and secretion of cellulolytic enzymes by $\delta$-sequences mediated multicopy integration of heterologous DNA sequences into the chromosomes of 
Saccharomyces cerevisiae. J. Ferment. Bioeng. 77: 468-473.

7. Cho KM, Yoo YJ, Kang HS. 1999. $\delta$-Integration of endo:exoglucanase and $\beta$-glucosidase genes into the yeast chromosomes for direct conversion of cellulose to ethanol. Enzyme Microb. Technol. 25: 23-30.

8. Lopes TS, Hakkaart GJ, Koerts BL, Raue HA, Planta RJ. 1991. Mechanism of high-copy-number integration of pMIRYtype vectors into the ribosomal DNA of Saccharomyces cerevisiae. Gene 105: 83-90.

9. Nieto A, Prieto JA, Sanz P. 1999. Stable high-copy-number integration of Aspergillus oryzae $\alpha$-amylase cDNA in an industrial baker's yeast strain. Biotechnol. Prog. 15: 459-466.

10. Kim YH, Sugiyama M, Yamagishi K, Kaneko Y, Fukui K, Kobayashi A, et al. 2005. A versatile and general splitting technology for generating targeted YAC subclones. Appl. Microbiol. Biotechnol. 69: 65-70.

11. Güldener U, Heck S, Fiedler T, Beinhauer J, Hegemann JH. 1996. A new efficient gene disruption cassette for repeated use in budding yeast. Nucleic Acids Res. 24: 2519-2524.

12. Kim YH, Ishikawa D, Ha HP, Sugiyama M, Kaneko $Y$, Harashima S. 2006. Chromosome XII context is important for rDNA function in yeast. Nucleic Acids Res. 34: 2914-2924.

13. Kim YH, Nam SW. 2010. Development of simultaneous YAC manipulation-amplification (SYMA) system by chromosome splitting technique harboring copy number amplification system. J. Life Sci. 20: 789-793.

14. Kim YH, Heo SY, Kim MJ, Lee JH, Kim YM, Nam SW. 2008. Optimal production of xylooligosaccharide by using recombinant endoxylanase from Bacillus subtilis. Korean J. Life Sci. 18: 52-57.

15. Lee LH, Kim DY, Han MK, Oh HW, Ham SJ, Park DS, et al. 2009. Characterization of an extracellular xylanase from Bacillus sp. HY-20, a bacterium in the gut of Apis mellifera. Korean J. Microbiol. 45: 332-338.

16. Chun YC, Jung KH, Lee JC, Park SH, Chung HK, Yoon KH. 1998. Molecular cloning and the nucleotide sequence of a Bacillus sp. KK-1 $\beta$-xylosidase gene. J. Microbiol. Biotechnol. 8: 28-33.

17. Sugiyama M, Nishizawa M, Hayashi K, Kaneko Y, Fukui K, Kobayashi A, et al. 2003. Repeated chromosome splitting targeted to delta sequences in Saccharomyces cerevisiae. J. Biosci. Bioeng. 96: 397-400.

18. Kim MJ, Kim BH, Nam SW, Choi ES, Shin DH, Cho HY, et al. 2013. Efficient secretory expression of recombinant endoxylanase from Bacillus sp. HY-20 in Saccharomyces cerevisiae. J. Life Sci. 23: 863-868.

19. Lee FW, Da Silva NA. 1997. Sequential delta-integration for the regulated insertion of cloned genes in Saccharomyces cerevisiae. Biotechnol. Prog. 13: 368-373. 